\title{
The Evaluation of Network Video QoE Based on an Improved Fuzzy Analytic Hierarchy Process
}

\author{
Min Yan ${ }^{1, a}$, Suixiang Gao ${ }^{1, b}$ and Wenguo Yang ${ }^{1, c}$ \\ ${ }^{1}$ University of Chinese Academy of Sciences 19 A Yuquan Rd, Shijingshan District, Beijing, \\ P.R.China \\ ayanmin910912@163.com, bsxgao@ucas.ac.cn, cyangwg@ucas.ac.cn
}

Keywords: network video QoE; improved FAHP; triangular fuzzy matrix correction.

Abstract. Network video (net-video) is universal with gradually development of network services. Most net-video providers can provide the same service and equipment. Thus the evaluation of quality of experience (QoE) becomes critical. Recent research has achieved to evaluate net-video QoE by some QoS parameters, with no subjective factors been considered, thus far from the definition of QoE. In this paper, we proposed an improved fuzzy analytic hierarchy process (IFAHP) based hierarchical structure that address the above shortcomings. A novel hierarchical structure was established, including objective and subjective factors of net-video QoE. IFAHP was proposed to evaluate net-video QoE based the hierarchical structure. IFAHP can check the consistency of fuzzy matrices and correct inconsistent triangle fuzzy matrix to a consistent one, meanwhile, maximize the retention of experts' information. At the end of this paper, a case study was given to evaluate net-video QoE.

\section{Introduction}

With the development of network and network services, network video (net-video) has played an important role in our daily life. More and more net-video providers arose. As most network video providers are able to provide the same services and equipment, the key point lies in the ability to provide superior net-video QoE. Consequently, net-video QoE, a new indicator of network services' quality, has been the focus of many net-video providers.Net-video QoE refers to the network quality, performance, and comfort that network users feel during watching the video. QoE is a measurement based on the satisfaction degree of users' experience. There are many related works for net-video QoE. The researches of $[1,2,3,4,5]$ only considered parameters of QoS and didn't take users' subjective feelings into consideration, far from the definition of QoE. However, fuzzy analytic hierarchy process (FAHP) can effectively combine subjective with objective factors of net-video QoE. FAHP is a method based on a hierarchical structure, easy for decision makers to make judgments on the quality of current net-video QoE, and also has a good effect on the evaluation of net-video QoE.

FAHP was developed by analytic hierarchy process and was applied in the evaluation of QoE. A method was proposed to evaluate QoE based on the service quality in wireless communication network in [6]. Gang Cheng et al. [7] gave a method to get evaluation of color ring back tone (CRBT) QoS, based on FAHP. Although the target goal was CRBT QoS, rather than QoE, the sub criteria contained customers' complaints, which indicating that users' subjective factors had been considered.

Because the elements of fuzzy matrix in FAHP are triangular fuzzy numbers rather than certain numbers, the traditional methods generating a weight vector from AHP can't be used in FAHP. Many researchers have put forward some new methods. Gang Cheng et al. [7] developed a formula to determine the relative importance of two indicators to generate weight vectors, but did not inspect the consistency of fuzzy matrix. Shukai Guo et al. [8] transformed a triangle fuzzy number to a real number by a formula proposed in their paper. In other words, they transformed a triangle fuzzy matrix to an ordinary one. Although they simplified the step, the missing of experts' information occurred at the same time. Wang L et al. [9] transformed the method of generating a weight vector to a nonlinear programming, with no need to rank fuzzy numbers. But they didn't give a method to correct the fuzzy matrix when it was inconsistent. The method to generate a weight vector in this paper overcomes these all disadvantages mentioned above. We inspected the consistency of triangle fuzzy matrix and 
corrected an inconsistent triangle fuzzy matrix to a consistent one while keeping experts' information to the maximum extent, to ensure the reliability of results.

There are two major contributions in this paper. Firstly, we built a novel hierarchical structure to evaluate net-video QoE which incorporates objective factors and subjective factors, consistent with the definition of QoE. Secondly, we proposed IFAHP method to give an evaluation of net-video QoE, based on the novel hierarchical structure, which can solve the problem of inaccurate or uncertain judgments of experts in AHP. To the best of our knowledge, this is also the first time that FAHP is applied to evaluate net-video QoE. The traditional method to generate weight vector by fuzzy judgment matrix was transformed into a nonlinear multi-objective programming in IFAHP, with no need to sort fuzzy numbers in traditional methods. And an inconsistent triangle fuzzy matrix can be corrected to a consistent one in IFAHP while keeping experts' information to the maximum extent.

The remainder of the paper is organized as follows. Firstly, we describe the establishment process of the hierarchical structure to evaluate net-video QoE. The subjective and objective indicators are also presented. Then we introduce the basic steps in the evaluation of net-video QoE based on FAHP. Thirdly, IFAHP is proposed in this paper. Forthly, we report the case study and results. Finally, we conclude this paper.

\section{A Novel Evaluation Hierarchical Structure of Net-video QoE}

The influence factors of net-video QoE can be divided into two categories: objective factors and subjective factors. The objective factors can be presented by network performance indicators. The subjective factors are closely related to users' complaints during watching a video.

Network performances are related to net-video QoE more or less. According to the research of [10], indicators that have a large degree of correlation with net-video QoE can be selected for the following six: delay, jitter, packet loss rate (PLR), error rate (ER), bandwidth and blocking rate (BR).

Subjective factors can be reflected by users' complaints when they are watching videos. We include five indicators contained which are shown in [11]. These five complaints are poor video access, too-long video access time, unsmooth video playback, video mosaic and asynchronous audio and video. They are also a large proportion of complaints that are received by net-video operators.

Combining subjective factors with objective factors of net-video QoE, a hierarchical structure to evaluate net-video QoE can be established as shown in Fig. 1.

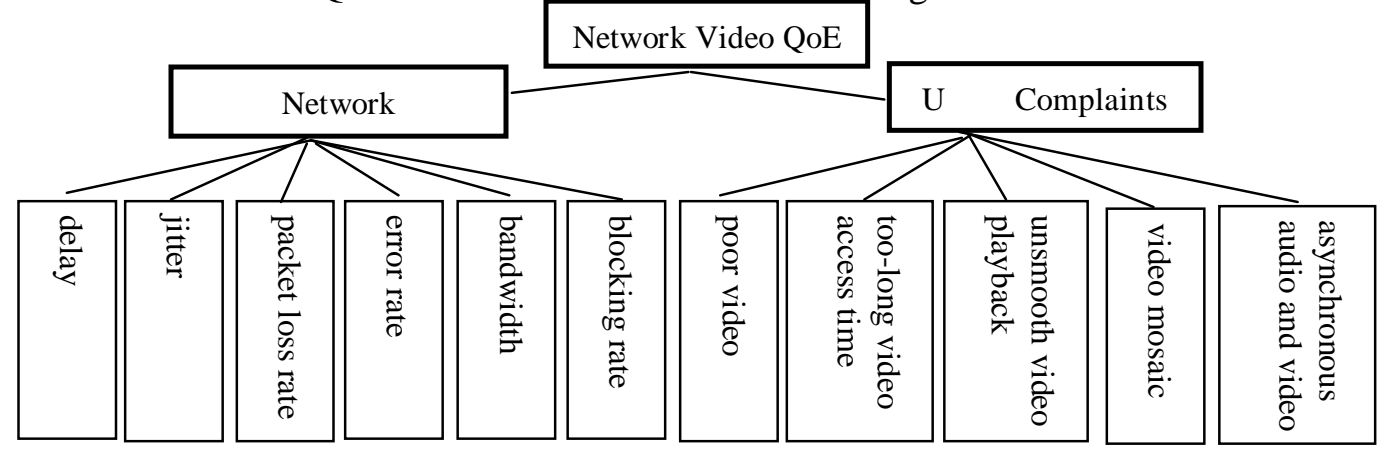

Fig. 1 The hierarchical structure of net-video QoE.

\section{Basic Steps to Evaluate Net-video QoE based on FAHP}

The application of FAHP in net-video QoE evaluation can be divided into five steps. Firstly, the hierarchical structure should be established, as what we do in the last section. Secondly, the fuzzy judgment matrices among each criterion layer and each sub criterion layer should be constructed, preparing for the ranking of priority among criteria and sub criteria. Thirdly, by the fuzzy judgment matrix, a normalized weight vector is generated, and the consistency of matrix is checked. In this paper, we proposed a new method to generate the weight vector, what will be described in next section. The fourth step of FAHP is scoring all sub criteria indicators, according to a scoring rule. Finally, we 
calculate the final score of net-video QoE, based on the weight vector and score of each indicator we get, and obtain the evaluation level of QoE of current net-video. All steps will be described in detail in the following content.

The constructing of fuzzy judgement matrix. Fuzzy judgment matrix is the matrix of triangular fuzzy numbers which is obtained by the comparison of two evaluation criteria. We assume fuzzy judgment matrix to be $R=\left(r_{i j}\right)_{n \times n}$, where $n$ is the number of evaluation criteria, and $r_{i j}$ is a triangular fuzzy number. We suppose $r_{i j}=\left(l_{i j}, m_{i j}, u_{i j}\right)$, where $l_{i j}, u_{i j}$ is the lower and upper bound of $r_{i j}$, respectively, and $m_{i j}$ is the median of the triangular fuzzy number. What need to be paid more attention to is that $m_{i j}$ $=\left(l_{i j}+u_{i j}\right) / 2$ is not always true. The value of each variable in $r_{i j}=\left(l_{i j}, m_{i j}, u_{i j}\right)$ is related to the relative importance of each two criteria in experts' opinions and $1 / 9 \leq l_{i j}<m_{i j}<u_{i j} \leq 9$ must be satisfied.

The obtain of normalized weight vector of criteria and sub criteria. The aim to obtain a normalized weight vector from fuzzy judgment matrix is to give a measure of relative importance among criteria and among sub criteria. To obtain a weight vector $\boldsymbol{w}=\left(w_{1}, w_{2}, \cdots, w_{n}\right)^{\mathrm{T}}$ from the fuzzy judgment matrix $R=\left(r_{i j}\right)_{n \times n}$, we need to ensure that the weight ratio between $w_{i}$ and $w_{j}$ can't exceed the range of triangle fuzzy number $r_{i j}=\left(l_{i j}, m_{i j}, u_{i j}\right)$, that is $l_{i j} \leq w_{i} / w_{j} \leq u_{i j}$. Moreover, the normalization of a weight vector is required to ensure that the sum of $w_{i}$ is 1 . In addition, we expect $w_{i} / w_{j}$ close to $m_{i j}$. We transformed the method to obtain a normalized weight vector from fuzzy judgment matrix into a nonlinear programming, same as [9]. The nonlinear programming model was shown in Eq.1.

$$
\begin{aligned}
& \max \lambda \\
& \text { s.t. }\left(m_{i j}-l_{i j}\right) \lambda w_{j}-w_{i}+l_{i j} w_{j} \leq 0, i=1, \mathrm{~L}, n-1, j=2, \mathrm{~L}, n, j>i, \\
& \quad\left(u_{i j}-m_{i j}\right) \lambda w_{j}+w_{i}-u_{i j} w_{j} \leq 0, i=1, \mathrm{~L}, n-1, j=2, \mathrm{~L}, n, j>i, \\
& \quad \sum_{l=1}^{n} w_{l}=1, w_{l}>0, l=1, \mathrm{~L}, n
\end{aligned}
$$

In Eq. $1, \lambda$ and $\boldsymbol{w}=\left(w_{1}, w_{2}, \cdots, w_{n}\right)^{\mathrm{T}}$ are all variables, $\left(l_{i j}, m_{i j}, u_{i j}\right)$ is the element of fuzzy judgment matrix. We can solve the nonlinear programming problem by genetic algorithm (GA), what will be described in detail in the next section.

What should be paid more attention is that the value $\lambda^{*}$ can be used as a criterion of the consistency degree of a fuzzy judgment matrix. If $\lambda^{*}$ is negative, the fuzzy judgment matrix is not consistent. If $\lambda^{*}$ equals 0 , it shows that the ratio $w_{i} / w_{j}$ just equals the lower bound $l_{i j}$ or upper bound $u_{i j}$ of the fuzzy number. What we expect mostly is $\lambda^{*}$ equals 1 , which symbolizes that the fuzzy judgment matrix is a fully consistent matrix and the ratio $w_{i} / w_{j}$ just equals $m_{i j}$, the median of triangle fuzzy number.

Based on the values of normalized weight vectors, the synthetic weight of each sub criterion to the goal can be calculated. Suppose that the weight of sub criterion $k$ to criterion $i$ is $w_{k i}$ and the weight value of criterion $i$ to the goal is $w_{i}$, we can obtain the synthetic weight of sub criterion $k$ to the goal by $w_{k i} \times w_{i}$. In order to facilitate the description in the next part, we sign the synthetic weight of sub criteria to the goal by $\mathbf{w}^{\prime}=\left(w_{1}{ }^{\prime}, w_{2}{ }^{\prime}, \cdots, w_{s}{ }^{\prime}\right)^{\mathrm{T}}$, where $s$ is the total number of sub criteria. Naturally, we are easy to know that the sum of all $w_{l}(l=1, \cdots, s)$ should be equal to 1 .

The score of sub criteria. After we get the synthetic weight of all sub criteria to the goal, the scoring of all sub criteria is needed to prepare for obtaining a score of net-video QoE. The scoring process of sub criterion can be divided into two parts: network performance and user complaints, according to the model of Fig.1.

Each sub criterion is generally divided into 3 to 4 levels. Each level has a score and the score range over 0 to 10 . Because of differences among the sensitivity of all network performance, the corresponding score varies. We can refer to the research of [10] for scoring. The score of network performance indicators can refer to the third table in [10]. As for the scoring of users'complaints, the difference only exists between receiving a complaint and without complaints for each users' complaint during them watching the video. Thus, the users'complaints are divided into two levels, 0 points and 10 points, standing for situations with complaints and without complaints, respectively. For facilitating the description, we sign the score of sub criterion $k$ as $P_{k}$. Accordingly, the scoring result of all sub criteria is $P=\left(P_{1}, P_{2}, \cdots, P_{s}\right)$. 
The score of net-video QoE. For an evaluation of net-video QoE, we assume that the synthetic weight of all sub criteria to the goal is $P=\left(P_{1}, P_{2}, \cdots, P_{s}\right)$ and the scoring result of sub criteria is $\mathbf{w}^{\prime}=\left(w_{1}{ }^{\prime}, w_{2}{ }^{\prime}, \cdots\right.$, $\left.w_{s}{ }^{\prime}\right)^{\mathrm{T}}$. Then we can calculate the score of net-video QoE by Eq.2:

$$
Q=\sum_{l=1}^{s} P_{i} \times w_{i}^{\prime}=P_{1} \times w_{1}^{\prime}+P_{2} \times w_{2}^{\prime}+\mathrm{L}+P_{s} \times w_{s}^{\prime} \text {. }
$$

The score $Q$ is also in the range [0,10]. We can map the score of the evaluation to a mean opinion score (MOS) standard, which is proposed by ITU in [12]. MOS model divided users' satisfaction into five levels: bad, poor, fair, good, excellent, according to the users' satisfaction of service. The mapping of evaluation score of QoE to five levels of MOS is uniform in the range of 0 to 10.

\section{The Improved Fuzzy Analytic Hierarchy Process (IFAHP)}

The method to generate weight vector from a fuzzy judgment matrix as mentioned above has a disadvantage. If the triangle fuzzy matrix is inconsistent, that is the optimal solution $\lambda^{*}<0$, how should we do to correct the matrix to a consistent one. For solving the problem, an improved FAHP method was proposed. Suppose that the original inconsistent triangle fuzzy matrix is $R$ and the consistent matrix after being corrected is $R^{\prime}$. We mark the element of $R^{\prime}$ as $r_{i j}{ }^{\prime}=\left(l_{i j}{ }^{\prime}, m_{i j}{ }^{\prime}, u_{i j}{ }^{\prime}\right)$. There are four conditions for $R^{\prime}$ to be satisfied. Firstly, $R^{\prime}$ should be consistent. Secondly, the numbers of the element of $R^{\prime}$ should also be in range $(0,9]$. Thirdly, the element of $R^{\prime}$ should satisfy $r_{j i}{ }^{\prime}=1 / r_{i j}{ }^{\prime}=\left(1 / u_{i j}{ }^{\prime}, 1 / l_{i j}{ }^{\prime}\right.$, $\left.1 / m_{i j}{ }^{\prime}\right)$ as same as $R$. Forthly, the difference between $R$ and $R^{\prime}$ should be as small as possible, to maximize the retention of experts' information.

To satisfy the four conditions above, the model in Eq.1 should be changed. For the first condition, what we only need to do is introducing $\lambda \geq 0$ to the model as a constraint condition. As for the second one, we can also introduce it as a constraint condition. For the third condition, we don't need to introduce any conditions to our model, because we only consider the element of the upper triangular matrix in our model. But to ensure the range of numbers in $r_{j i}{ }^{\prime}$ is also $(0,9]$, we need to change the range of numbers in $r_{i j}{ }^{\prime}$ to $[1 / 9,9]$, rather than $(0,9]$. As for the last condition, we can introduce a deviation function to measure the degree of modification. The deviation function is defined as Eq.3:

$$
\sum_{i, j}\left(l_{i j}^{\prime}-l_{i j}\right)^{2}+\left(m_{i j}{ }^{\prime}-m_{i j}\right)^{2}+\left(u_{i j}^{\prime}-u_{i j}\right)^{2} .
$$

The value of the deviation function is the less the better. Therefore it can be added to the objective functions. Thus we change our model as Eq.4.

$$
\begin{aligned}
& \max \lambda \\
& \max -\sum_{i, j}\left(l_{i j}^{\prime}-l_{i j}\right)^{2}+\left(m_{i j}^{\prime}-m_{i j}\right)^{2}+\left(u_{i j}^{\prime}-u_{i j}\right)^{2} \\
& \text { s.t. }\left\{\begin{array}{l}
\left(m_{i j}^{\prime}-l_{i j}^{\prime}\right) \lambda w_{j}-w_{i}+l_{i j}^{\prime} w_{j} \leq 0, i=1, \mathrm{~L}, n-1, j=2, \mathrm{~L}, n, j>i, \\
\left(u_{i j}^{\prime}-m_{i j}^{\prime}\right) \lambda w_{j}+w_{i}-u_{i j}^{\prime} w_{j} \leq 0, i=1, \mathrm{~L}, n-1, j=2, \mathrm{~L}, n, j>i, \\
\sum_{l=1}^{n} w_{l}=1, w_{l}>0, l=1, \mathrm{~L}, n, \\
\lambda \geq 0 \\
1 / 9 \leq l_{i j}^{\prime}<m_{i j}^{\prime}<u_{i j}^{\prime} \leq 9, \quad i=1, \mathrm{~L}, n-1, j=2, \mathrm{~L}, n, j>i,
\end{array}\right.
\end{aligned}
$$

The improved model in Eq.4 is a MONP problem and one of its' objective functions is discontinuous. As we know, GA shows great effect on solving discontinuous and multi goals problems. Therefore, GA is used to solving the improved model in Eq.4. GA mainly includes six steps: coding, initial population generation, adaptive value evaluation, selection, crossover and mutation. Due to the limitation of space, it won't be introduced in detail in this paper, but you can refer to [13] to know more about GA.

The most crucial step in GA is the choice of fitness function. We'll combine the objective functions with the constraint conditions of our new model in Eq.4 to construct the fitness function. Firstly, we 
transform the multi objective programming into a single objective programming problem by linear weighting method as Eq.5.

$$
\max \left[\sigma_{1} \cdot \lambda-\sigma_{2} \sum_{i, j}\left(l_{i j}^{\prime}-l_{i j}\right)^{2}+\left(m_{i j}^{\prime}-m_{i j}\right)^{2}+\left(u_{i j}^{\prime}-u_{i j}\right)^{2}\right] \text {. }
$$

As for the constraint conditions, penalty function is introduced to transform the constrained model into an unconstrained optimization problem. In summary, the conversion of the model in Eq.4 is

$$
\min \left[-\sigma_{1} f_{1}+\sigma_{2} f_{2}+\gamma_{1} g_{1}+\gamma_{2} g_{2}+\gamma_{3} g_{3}+\gamma_{4} g_{4}\right] \text {. }
$$

Where

$$
\begin{aligned}
& f_{1}=\lambda, f_{2}=\sum_{i, j}\left(l_{i j}{ }^{\prime}-l_{i j}\right)^{2}+\left(m_{i j}{ }^{\prime}-m_{i j}\right)^{2}+\left(u_{i j}^{\prime}-u_{i j}\right)^{2} . \\
& g_{1}=\left|\sum_{l=1}^{n} w_{l}-1\right|, g_{2}=\sum_{i, j}\left(\lambda-\mu\left(w_{i} / w_{j}\right)\right), g_{3}=\sum_{i, j}\left(m_{i j}{ }^{\prime}-l_{i j}^{\prime}\right), g_{4}=\sum_{i, j}\left(u_{i j}^{\prime}-m_{i j}^{\prime}\right) .
\end{aligned}
$$

The constraint condition $w_{l}>0$ and the range of $\left(l_{i j}{ }^{\prime}, m_{i j}{ }^{\prime}, u_{i j}{ }^{\prime}\right)$ can be added in the range of variable values in GA. Thus, the new objective function can be used as a fitness function. So the fitness function of GA in our new model is

$$
\text { fitness }=-\sigma_{1} f_{1}+\sigma_{2} f_{2}+\gamma_{1} g_{1}+\gamma_{2} g_{2}+\gamma_{3} g_{3}+\gamma_{4} g_{4} \text {. }
$$

Where $f_{1}, f_{2}, g_{1}, g_{2}, g_{3}, g_{4}$ are defined as Eq.8.

The implementation of GA in this paper uses GAOT, which is widely used as a toolbox of MATLAB. In GAOT, the fitness function is defined as the above fitness and the crossover rate and mutation rate are randomly generated within their range.

\section{Case Study}

According to the opinion of some experts, the hierarchical structure of Fig. 1 is reasonable. Moreover, through the result of a questionnaire, we get the triangle fuzzy matrix among network performance indexes and users' complaints as shown in Table 1 and Table 2, respectively. Based on Table 1 and Table 2, we calculate that $\lambda^{*}=10^{-4} \approx 0$ and $\lambda^{*}=0.0087>0$, respectively, by the improved model in Eq.4. Thus we know that the fuzzy matrix in Table 1 is inconsistent and needs to be corrected. The correcting result of Table 1 is shown in Table 3, by solving the model in Eq.4. From Table 3 we can obtain the optimal solution of the corrected fuzzy matrix is that $\mathbf{w 1}=\left(w_{1}, w_{2}, \cdots, w_{6}\right)^{\mathrm{T}}=$ $(0.3291,0.2365,0.0874,0.0534,0.2088,0.0848)^{\mathrm{T}}, \lambda^{*}=0.022$. And because of the value of $\lambda^{*}$ of Table 2 is positive, the fuzzy matrix in Table 2 is consistent and needn't to be corrected, we can get the normalized weight vector of user complaints directly by solving the model in Eq. 1 , as $\mathbf{w} 2=\left(w_{1}, w_{2}, \cdots\right.$, $\left.w_{5}\right)^{\mathrm{T}}=(0.2558,0.3698,0.1258,0.1249,0.1236)^{\mathrm{T}}$. We can also get the triangle fuzzy matrix among criteria by the same way. The fuzzy matrix is shown in Table 4 and the result calculated by model in Eq. 1 is that $\mathbf{w 3}=\left(w_{1}, w_{2}\right)^{\mathrm{T}}=(0.34,0.66)^{\mathrm{T}}, \lambda^{*}=1$. Because $\lambda^{*}$ equals 1 , we know that the fuzzy matrix of Table 4 is a fully consistent matrix.

Based on the results above, the synthetic weight of sub criteria of network performance indexes is that $\mathbf{w} 1^{\prime}=\left(w_{1}{ }^{\prime}, w_{2}{ }^{\prime}, \cdots, w_{6}{ }^{\prime}\right)^{\mathrm{T}}=(0.1119,0.0804,0.0297,0.0182,0.071,0.0288)^{\mathrm{T}}$. And the synthetic weight of sub criteria of users' complaints is that $\mathbf{w} \mathbf{2}^{\prime}=\left(w_{1}{ }^{\prime}, w_{2}{ }^{\prime}, \cdots, w_{5}{ }^{\prime}\right)^{\mathrm{T}}=(0.1688,0.2441$, $0.083,0.0824,0.0816)^{\mathrm{T}}$.

If we monitor the current network and get all the parameters we need, we can calculate the score of net-video QoE. Suppose that delay is $125 \mathrm{~ms}$, jitter is $45 \mathrm{~ms}$, package loss rate is $2 \times 10^{-5}$, error rate is $1 \times 10^{-6}$, bandwidth is $2.5 \mathrm{Mbps}$, blocking rate is $1 \%$, and suppose that we only get a user complaint of too-long video access time. Then we can calculate the score of net-video QoE by $Q=(8,10,7,10$, $10,8) \cdot \mathbf{w} \mathbf{1}^{\prime}+(10,0,10,10,10) \cdot \mathbf{w} \mathbf{2}^{\prime}=7.1875$. So the scoring result of current network is 7.1875 , corresponding to "good" as the MOS standard. 
Table 1. The triangle fuzzy matrix among network performance indexes

\begin{tabular}{|l|l|l|l|l|l|l|}
\hline Indexes & Delay & Jitter & PLR & ER & Bandwidth & BR \\
\hline Delay & $(1,1,1)$ & $(1 / 6,1 / 5,1 / 4)$ & $(1 / 8,1 / 7,1 / 6)$ & $(1 / 8,1 / 7,1 / 6)$ & $(1 / 2,1,2)$ & $(1 / 2,1,2)$ \\
\hline Jitter & $(4,5,6)$ & $(1,1,1)$ & $(1 / 4,1 / 3,1 / 2)$ & $(1 / 4,1 / 3,1 / 2))$ & $(1 / 2,1,2)$ & $(1 / 2,1,2)$ \\
\hline PLR & $(6,7,8)$ & $(2,3,4)$ & $(1,1,1)$ & $(1 / 4,1 / 3,1 / 2)$ & $(1 / 2,1,2)$ & $(1 / 2,1,2)$ \\
\hline ER & $(6,7,8)$ & $(2,3,4)$ & $(2,3,4)$ & $(1,1,1)$ & $(2,3,4)$ & $(2,3,4)$ \\
\hline Bandwidth & $(1 / 2,1,2)$ & $(1 / 2,1,2)$ & $(1 / 2,1,2)$ & $(1 / 4,1 / 3,1 / 2)$ & $(1,1,1)$ & $(1 / 2,1,2)$ \\
\hline BR & $(1 / 2,1,2)$ & $(1 / 2,1,2)$ & $(1 / 2,1,2)$ & $(1 / 4,1 / 3,1 / 2)$ & $(1 / 2,1,2)$ & $(1,1,1)$ \\
\hline
\end{tabular}

Table 2. The triangle fuzzy matrix among users' complaints

\begin{tabular}{|l|l|l|l|l|l|}
\hline User complaints & Poor video access & $\begin{array}{l}\text { Too-long video } \\
\text { access time }\end{array}$ & $\begin{array}{l}\text { Unsmooth video } \\
\text { playback }\end{array}$ & Video mosaic & $\begin{array}{l}\text { Asynchronous } \\
\text { audio and video }\end{array}$ \\
\hline Poor video access & $(1,1,1)$ & $(1 / 2,1,2)$ & $(2,3,4)$ & $(2,3,4)$ & $(1,2,3)$ \\
\hline $\begin{array}{l}\text { Too-long video } \\
\text { access time }\end{array}$ & $(1 / 2,1,2)$ & $(1,1,1)$ & $(2,3,4)$ & $(2,3,4)$ & $(1,2,3)$ \\
\hline $\begin{array}{l}\text { Unsmooth video } \\
\text { playback }\end{array}$ & $(1 / 4,1 / 3,1 / 2)$ & $(1 / 4,1 / 3,1 / 2)$ & $(1,1,1)$ & $(1 / 2,1,2)$ & $(1,2,3)$ \\
\hline Video mosaic & $(1 / 4,1 / 3,1 / 2)$ & $(1 / 4,1 / 3,1 / 2)$ & $(1 / 2,1,2)$ & $(1,1,1)$ & $(1,2,3)$ \\
\hline $\begin{array}{l}\text { Asynchronous } \\
\text { audio and video }\end{array}$ & $(1 / 3,1 / 2,1)$ & $(1 / 3,1 / 2,1)$ & $(1 / 3,1 / 2,1)$ & $(1 / 3,1 / 2,1)$ & $(1,1,1)$ \\
\hline
\end{tabular}

Table 3. The corrected triangle fuzzy matrix among network performance indexes

\begin{tabular}{|l|l|l|l|l|l|l|}
\hline Indexes & Delay & Jitter & PLR & ER & Bandwidth & BR \\
\hline Delay & $(1,1,1)$ & $(0.17,0.2,1.42)$ & $(0.13,0.14,3.85)$ & $(0.13,0.14,6.29)$ & $(0.5,1,2)$ & $(0.5,1,3.95)$ \\
\hline Jitter & $(0.71,5,6)$ & $(1,1,1)$ & $(0.25,0.33,4.35)$ & $(0.25,0.33,4.52)$ & $(0.5,1,2)$ & $(0.5,1,2.83)$ \\
\hline PLR & $(0.26,7,8)$ & $(0.23,3,4)$ & $(1,1,1)$ & $(0.25,0.33,1.67)$ & $(0.41,1,2)$ & $(0.5,1,2)$ \\
\hline ER & $(0.16,7,8)$ & $(0.22,3,4)$ & $(0.6,3,4)$ & $(1,1,1)$ & $(0.19,3,4)$ & $(0.58,3,4)$ \\
\hline Bandwidth & $(0.5,1,2)$ & $(0.5,1,2)$ & $(0.5,1,2.5)$ & $(0.25,0.33,5.15)$ & $(1,1,1)$ & $(0.5,1,2.5)$ \\
\hline BR & $(0.25,1,2)$ & $(0.35,1,2)$ & $(0.5,1,2)$ & $(0.25,0.33,1.73)$ & $(0.4,1,2)$ & $(1,1,1)$ \\
\hline
\end{tabular}
Table 4 . The corrected triangle fuzzy matrix among criteria

\begin{tabular}{|l|l|l|}
\hline Indexes & Network performance indexes & User complaints \\
\hline Network performance indexes & $(1,1,1)$ & $(1 / 3,1 / 2,1)$ \\
\hline User complaints & $(1,2,3)$ & $(1,1,1)$ \\
\hline
\end{tabular}

\section{Conclusions}

This paper solves a critical problem that how to combine subjective factors into the evaluation of net-video QoE. To address this problem, we proposed a novel hierarchical structure, which combines subjective and objective factors to guarantee the rationality of evaluation. Meanwhile, IFAHP was proposed to evaluate net-video QoE, based on the hierarchical structure, to solve the problem of inaccurate or uncertain judgments of experts in AHP. IFAHP can correct an inconsistent fuzzy matrix to a consistent one, with the maximum retention of experts' information. Scoring and case study were carried out to evaluate net-video QoE using proposed IFAHP, the results show that delay and the access time of net-video should be regarded as main indexes to be improved for net-video providers. Besides, IFAHP has strong applicability, thus we can consider extending its application to the evaluation of QoE for other fields in the future.

\section{Acknowledgements}

This work was supported by the National 973 Plan project under Grant No.2011CB706900, the National 863 Plan project under Grant No.2011AA01A102, the NSFC (11331012, 71171189, 1157 1015), the "Strategic Priority Research Program" of Chinese Academy of Sciences (XDA06010302) .

\section{References}

[1] WU Ji-xiang, LI Fan,XIA Jing-bo et al., QoE evaluation method through generalized linear model, Application Reaserrch of Computers, 2012, 29(10): 3911-3913.(In Chinese) 
[2] ZHANG Dalu, ZHU Jiaqi. QoE evaluation model for IPTV in network transmission. Computer Engineering and Applications,2013, 49(20): 71-76. (In Chinese)

[3] Dai Q. A survey of quality of experience[M]//Energy-Aware Communications. Springer Berlin Heidelberg, 2011: 146-156.

[4] Serral-Gracià R, Cerqueira E, Curado M, et al. An overview of quality of experience measurement challenges for video applications in IP networks[M]//Wired/Wireless Internet Communications. Springer Berlin Heidelberg, 2010: 252-263.

[5] Hui Zhang,Conviva. Big Data Differentiators A QoE Predictive Model For Video SDN.2014

[6] Du Y, Zhou W, Chen B. A QoE based evaluation of service quality in wireless communication network[C]//New Trends in Information and Service Science, 2009. NISS'09. International Conference on. IEEE, 2009: 552-557.

[7] CHENG Gang, LIAO Jian-xin, ZHU Xiao-min et al., CRBT QoS evaluation based on triangle fuzzy number analytic hierarchy process, Journal of Chongqing University of Posts and Telecommunications(Natural Science Edition), 2008, 20(1): 94-99. (In Chinese)

[8] GUO Shukai,TIAN Liqin,SHEN Xueli.Research on FAHP method in user behaviour trust computation.Computer Engineering and Applications, 2011，47(12): 59-61. (In Chinese)

[9] Wang L, Chu J, Wu J. Selection of optimum maintenance strategies based on a fuzzy analytic hierarchy process[J]. International Journal of Production Economics, 2007, 107(1): 151-163.

[10] Kim H J, Lee D H, Lee J M, et al. The QoE evaluation method through the QoS-QoE correlation model[C]//Networked Computing and Advanced Information Management, 2008. NCM'08. Fourth International Conference on. IEEE, 2008, 2: 719-725.

[11]JIN Yu,LI Xiao-lin, LI Xue-song, Quality of Experience Assessment Model and KQI Weigh Calculation Method, Computer Engineering, 2013,39(2). (In Chinese)

[12]ITU Mean opinion score(MOS)terminology,telecommunication standardization sector 2003.

[13]ZHANG Wen-xiu, LIANG Yi, Mathmatical foundation of genetic algorithm, Xi'an Jiao Tong University press. (In Chinese) 